\title{
ER-Golgi transport: authors' response
}

\author{
Federica Brandizzi and Charles Barlowe
}

We appreciate the comments on our Review article (Organization of the ER-Golgi interface for membrane traffic control. Nature Rev. Mol. Cell Biol. 14, 382-392 (2013)) $)^{1}$, by Alexandre A. Mironov (ER-Golgi transport could occur in the absence of COPII vesicles. Nature Rev. Mol. Cell Biol. http://dx.doi. org/10.1038/nrm3588-c1 (2014)) $)^{2}$ regarding COPII-independent transport between the ER and Golgi. Of course we agree that there are open questions regarding the pathways and machinery that underlie trafficking in the early secretory system. Given length limitations, we chose to focus our Review ${ }^{1}$ on components (including coat protein complex II (COPII)) that are well documented to influence trafficking and the endoplasmic reticulum (ER)-Golgi interface in many cell types. The Review ${ }^{1}$ was not about whether specific COPII subunits are functionally required on all anterograde transport carriers in all cells.

We encourage the reader to fully explore the literature cited in the Correspondence ${ }^{2}$, in the context of many reports demonstrating a role for coat complexes in bidirectional transport between the ER and Golgi. We also caution the reader when interpreting negative results. For example, a failure to detect short-lived intermediates by microscopy is not necessarily evidence of their absence. Moreover, some of the points in the Correspondence ${ }^{2}$ represent selected findings from these published works and do not accurately reflect conclusions from the current literature. For instance, it is suggested that a condition in which budding yeast can survive in the absence of the Sec13 subunit of COPII ${ }^{3}$ indicates that COPII is not needed for transport through the early secretory pathway. However, it is well supported and it has been recently shown ${ }^{4}$ that under this condition COPII coats are still very much involved in generating transport intermediates, although the rigidity of the coat is altered.

Finally, the good news is that there is clearly much more experimentation ahead to fully understand the molecular structure and function of transport carriers in this important area of membrane biology.

$$
\begin{array}{r}
\text { Federica Brandizzi is at the DOE Plant Research } \\
\text { Laboratory and Department of Plant Biology, } \\
\text { Michigan State University, East Lansing, } \\
\text { Michigan 48824, USA. } \\
\text { Charles Barlowe is at the Department of } \\
\text { Biochemistry, Dartmouth Medical School, } \\
\text { Hanover, New Hampshire 03755, } \\
\text { USA. } \\
\text { Correspondence to C.B. } \\
\text { e-mail: charles.barlowe@dartmouth.edu } \\
\text { doi: 10.1038/nrm3588-c2 }
\end{array}
$$

1. Brandizzi, F. \& Barlowe, C. Organization of the ER-Golgi interface for membrane traffic control. Nature Rev. Mol. Cell Biol. 14, 382-392 (2013).

2. Mironov, A. A. ER-Golgi transport could occur in the absence of COPII vesicles. Nature Rev. Mol. Cell Biol. http://dx.doi.org/10.1038/nrm3588-c1 (2014).

3. Elrod-Erickson, M. J. \& Kaiser, C. A. Genes that control the fidelity of endoplasmic reticulum to Golgi transport identified as suppressors of vesicle budding mutations. Mol. Biol. Cell 7, 1043-1058 (1996).

4. Copic, A. et al. ER cargo properties specify a requirement for COPII coat ridigity mediated by Sec13p. Science 335, 1359-1362 (2012).

Competing interests statement

The authors declare no competing interests. 